\section{A therapeutic genome editing primer for cardiologist}

\author{
Martina Caiazza, Daniele Masarone, \\ Giuseppe Limongelli \\ Department of Cardiology, Università \\ Degli Studi Della Campania, Luigi \\ Vanvitelli, Naples, Italy
}

\begin{abstract}
Genome editing, or genome engineering is a type of genetic engineering in which DNA is inserted, deleted or replaced in the genome of a living organism using engineered nucleases, or molecular scissors. Genome editing is being rapidly adopted into all fields of biomedical research, including the cardiovascular field, where it has facilitated a greater understanding of lipid metabolism, electrophysiology, cardiomyopathies, and other cardiovascular disorders, has helped to create a wider variety of cellular and animal models, and has opened the door to a new class of therapies. In this review, we discuss the applications of in vivo genome-editing therapies for cardiovascular disorder.
\end{abstract}

\section{Introduction}

Genome editing, or genome engineering is a type of genetic engineering in which DNA is inserted, deleted or replaced in the genome of a living organism using engineered nucleases, or molecular scissors. ${ }^{1}$ Genome editing is being rapidly adopted into all fields of biomedical research, including the cardiovascular field, where it has facilitated a greater understanding of lipid metabolism, electrophysiology, cardiomyopathies, and other cardiovascular disorders, has helped to create a wider variety of cellular and animal models, and has opened the door to a new class of therapies. ${ }^{2}$ In this review, we discuss the applications of in vivo genome-editing therapies for cardiovascular disorder.

\section{Genome editing tools}

Currently, the three most commonly used genome-editing tools are zinc finger nucleases (ZFNs), transcription activatorlike effector (TALEs), and clustered regularly interspaced short palindromic repeats CRISPR-associated 9 (Cas9) systems, all of which induce double-strand DNA breaks to achieve genome editing.

ZFNS: are engineered restriction enzymes adapted from zinc finger-containing transcription factors - attached to the endonuclease domain of the bacterial FokI restriction enzyme. ${ }^{3}$ Each zinc finger domain recognizes a 3- to 4-bp DNA sequence, and tandem domains can potentially bind to an extended nucleotide sequence that is unique within a cell's genome. To cleave a specific site in the genome, ZFNs are designed as a pair that recognizes two sequences flanking the site, one on the forward strand and the other on the reverse strand. Upon binding of the ZFNs on either side of the site, the pair of FokI domains dimerize and cleave the DNA at the site, generating a double-strand break (DSB) with 5' overhangs. ${ }^{3}$ Cells repair DSBs using either non homologous end joining (NHEJ), which can occur during any phase of the cell cycle, but occasionally results in erroneous repair, or homologydirected repair (HDR), which typically occurs during late $S$ phase or $G_{2}$ phase when a sister chromatid is available to serve as a

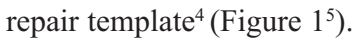

TALEs: first identified in Xanthomonas bacteria, are naturally occurring or artificially designed proteins that modulate gene transcription. ${ }^{6}$ These proteins recognize and bind DNA sequences based on a variable numbers of tandem repeats. Each repeat is comprised of a set of $\square 34$ conserved amino acids; within this conserved domain, there are usually two amino acids that distinguish one TALE from another. Interestingly, TALEs have revealed a simple cipher for the one-to-one recognition of proteins for DNA bases. Synthetic TALEs have been used to successfully target genes in a variety of species, including humans ${ }^{7}$ (Figure $2^{8}$ ).

CRISPR-Cas9: is a genome editing tool that is creating a buzz in the science world. It is faster, cheaper and more accurate than previous techniques of editing DNA and has a wide range of potential applications. ${ }^{9}$ CRISPR-Cas9 is a unique technology that enables geneticists and medical researchers to edit parts of the genome by removing, adding or altering sections of the DNA sequence. It is currently the simplest, most versatile and precise method of genetic manipulation. The CRISPR-Cas9 system consists of two key molecules that introduce a mutation into the DNA. There is an enzyme called Cas9, which acts as a pair of molecular scissors that can cut the two strands of DNA at a specific location in the genome so that bits of DNA can then be added or removed. ${ }^{9}$ Then there is a guide RNA (gRNA).

The scaffold part of this binds to DNA and the pre-designed sequence guides Cas9
Correspondence: Martina Caiazza, Department of Cardiology, Università Degli Studi Della Campania, Luigi Vanvitelli, Naples, Italy.

E-mail: martina.caiazza@yahoo.it

Key words: Therapeutic genome editing; cardiovascular disease.

Received for publication: 27 December 2017. Revision received: 15 March 2018.

Accepted for publication: 24 April 2018.

This work is licensed under a Creative Commons Attribution NonCommercial 4.0 License (CC BY-NC 4.0).

(C) Copyright M. Caiazza et al., 2018

Licensee PAGEPress, Italy

Cardiogenetics 2018; 8:7262

doi:10.4081/cardiogenetics.2018.7262

to the right part of the genome. This makes sure that the Cas9 enzyme cuts at the right point in the genome. The guide RNA is designed to find and bind to a specific sequence in the DNA.

The guide RNA has RNA bases that are complementary to those of the target DNA sequence in the genome. This means that, at least in theory, the guide RNA will only bind to the target sequence and no other regions of the genome. ${ }^{10}$ The Cas9 follows the guide RNA to the same location in the DNA sequence and makes a cut across both strands of the DNA. At this stage the cell recognizes that the DNA is damaged and tries to repair it (Figure 3).

\section{Therapeutic genome editing}

Genome editing based therapy can be achieved through a number of approaches including correction or inactivation of deleterious mutations, addition of therapeutic transgenes, or disruption of viral DNA. Pathogenic mutations can be broadly classified as gain or loss-of-function.

An example of gain-of-function mutation is E76K mutation in PTPN11 gene, which encodes the protein tyrosine phosphatase 11 (SHP2). Several gain-of-function mutations in PTPN11 have been identified in human hematopoietic malignancies and solid tumors. In addition, the mutation rate for SHP2 is the highest for colorectal cancer (CRC) among solid tumors. ${ }^{11}$ These diseases may be corrected by using NHEJmediated induced mutations to specifically inactivate the mutant gene while leaving the wild type copy intact on the other allele. For gain of function mutations, such as the 
$A B C A 1$ for familial hypercholesterolemia HDR could be used to change the gain-offunction allele to the wild type sequence, recovering gene function and eliminating pathogenic activity while preserving physiological levels of gene expression. ${ }^{12}$

For deleterious loss of function mutations and protective gain of function mutations, a therapeutic effect may also be achieved by introducing a copy of the wild type gene or gain-of-function mutant respectively. Gene insertion may also be used to stably confer cells novel functions that protect against disease. Such gene insertion strategies are similar to viralmediated gene therapy, but with the advantage of providing better control over transgene copy number and expression levels, which may be important for gene targets whose function is sensitive to expression levels. ${ }^{13}$

Therapeutic genome editing approaches can be divided into two categories: i) ex vivo editing of stem or progenitor cells which are delivered into the patient; ii) direct in vivo administration, either locally or systemically, of gene editing components into the patient.

Given the success of genome modification achieved in a range of stem, progenitor and primary cells, as well as progress of the ongoing HIV trial, it is likely that the former approach will continue to represent the low hanging fruit for this technology. ${ }^{14}$ While published proof-of-concept studies using the second approach (direct delivery of genome editing components) in animal disease models are few in number, they provide reasons to be optimistic that, with the appropriate delivery technology, these types of therapeutics can be developed. In evaluating the feasibility of a genome editing based therapy, the therapeutic effect of the desired genetic change should first be clearly established.

Subsequently, the success of a given strategy will depend on the ease with which a therapeutic modification threshold is achieved, a criteria that is governed by the fitness of edited cells, the DSB repair pathway utilized to edit the genome, and the efficiency of delivery of genome editing molecules to target cell types. ${ }^{13}$

\section{Therapeutic genome editing and cardiovascular disease}

In vivo genome editing could potentially be used to correct disease-causing mutations by HDR in the presence of a repair template. Although no such approach has yet been reported for a genetic disorder affecting the cardiovascular system, proofof-principle studies in mice have been reported for hereditary tyrosinemia type I and ornithine transcarboxylase deficiency. ${ }^{15}$ The first example of in vivo gene editing with CRISPR/Cas9 that involved the correction of a mouse model of hereditary tyrosinemia type I following hydrodynamic tail vein injection of plasmid DNA into mice. Although overall gene-editing efficiencies were relatively low, it was possible to demonstrate correction of the disease phenotype with this experiment. Although the method is likely not translatable to humans, this study was a landmark in demonstrating in vivo gene editing with CRISPR/Cas9 in adult tissues. ${ }^{16}$

Two categories of cardiovascular dis-

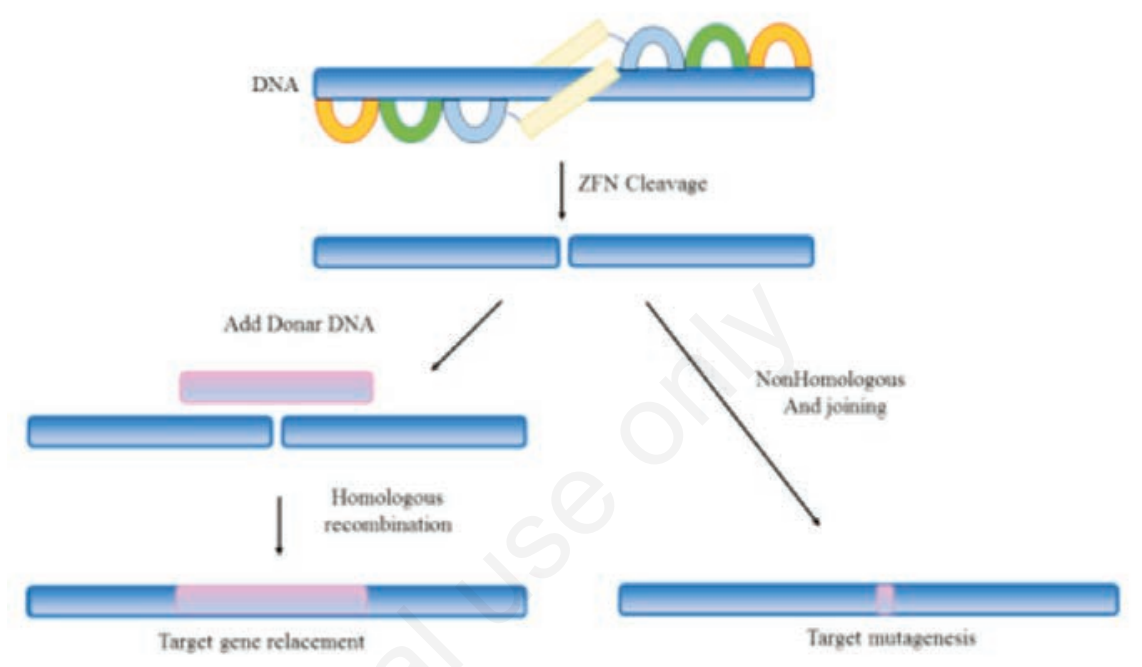

Figure 1. Repair outcomes of a genomic double-strand break, illustrated for the case of ZFN cleavage. A pair of three-finger ZFNs is shown at the top in association with a target gene. If a homologous donor DNA is provided repair can proceed by homologous recombination using the donor as template. Alternatively, the break can be repaired by nonhomologous end joining, leading to mutations at the cleavage site. $^{5}$

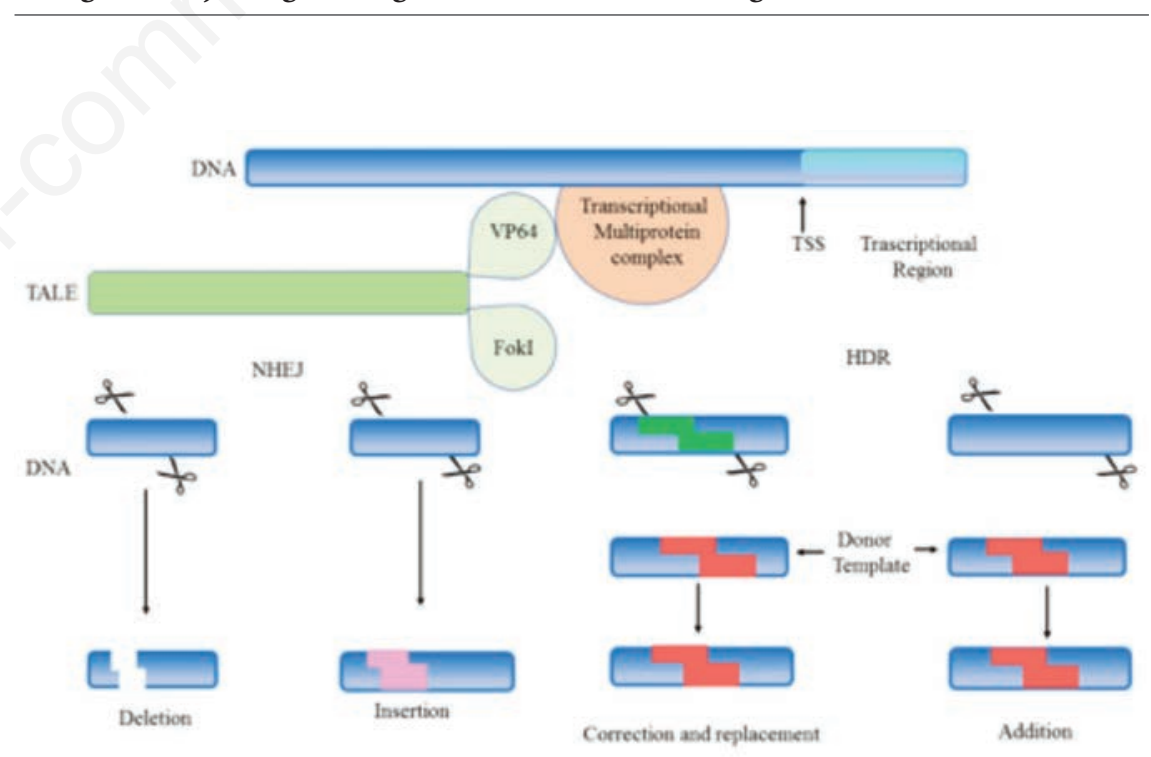

Figure 2. The TALE DNA-binding domain is fused to the synthetic VP64 transcriptional activator and assembles transcriptional multiprotein complexes with RNA polymerase II. TALE nucleases (TALENs)-induced DNA double-strand breaks (DSBs) typically result in one of two major DNA damage repair pathways: nonhomologous end joining (NHEJ) or homologous directed recombination (HDR) repair. NHEJ directly ligates the ends of the broken DNA strands and introduces an error-prone deletion or insertion at the repair site by DNA repair proteins and ligase. Gene editing through HDR from the donor template can result in gene additions and replacements. ${ }^{8}$ 
eases are the most amenable to an in vivo genome-editing therapeutic approach: i) Inherited cardiomyopathies: are caused by single dominant mutations within sarcomere genes. In principle, these diseases could be prevented or arrested both by correction of the mutant allele by HDR, or specific disruption of the mutant allele by NHEJ, removing the dominant influence of the mutant allele. The first strategy might be extremely challenging to achieve owing to limited HDR in adult cardiomyocytes, conversely an approach similar to the second strategy has been achieved in a mouse model through the use of RNA interference, suggesting the viability of the NHEJ genome-editing approach; ${ }^{17} \quad$ ii) Dyslipidemias: PCSK9, a protein that tion of LDL clearance, has recently emerged as a promising protein and genetic target. Naturally occurring PCSK9 loss-offunction mutations not only reduce blood LDL-C levels but also reduce coronary heart disease risk. ${ }^{18}$ Multiple studies have showed that inhibitors of PCSK9 through antibody targeting or antisense oligonucleotide silencing reduce blood LDL-C levels and, ultimately, atherosclerotic vascular disease. ${ }^{19}$ These therapeutic approaches have started to yield clinical success, but as with statin drugs, they have the substantial limitation of needing to be taken at repeated intervals over the course of a patient's lifetime to obtain the desired therapeutic benefit. increases plasma LDL-C levels via inhibi-
In light of these observations, genome editing of PCSK9 offers a highly attractive alternative approach as it could significantly reduce coronary heart disease risk while with only a single treatment. ${ }^{19}$ Recent work has described the ability to permanently disrupt PCSK9 with high efficiency using the CRISPR/Cas9 system in vivo. ${ }^{20}$ Targeted genome editing, in theory, is capable of modifying any endogenous gene sequence of interest; this can be performed in cells or organisms, and may be applied to clinical gene-based therapies in the future. With current technologies, highly accurate, specific, and reliable gene editing cannot be achieved.

\section{Conclusions}

Given the accelerating pace of technological advances and broad range of basic science and clinical applications, genome editing presents a lot of opportunities for several patients with different diseases. The extraordinary progress made over the past few years to improve efficacy, specificity and delivery across the major genome editing platforms provides hope that the true promise of this groundbreaking technology can be realized. Despite being in its infancy, genome editing presents tantalizing opportunities for tackling a number of diseases that are beyond the reach of previous theraalso offering a lifelong therapeutic effect

pies. $^{21}$

In the next decade there will be tens of genome editing-based clinical trials that will be developed by academics, biotechnology start-up and pharmaceutical companies.

Nonetheless, there remain important issues to be resolved.

There is a need to develop safe and effective mechanisms to deliver the genome-editing machinery to a wide variety of tissues in vivo. ${ }^{22}$

Finally, a flexible and adaptive regulatory framework needs to be developed to take into account the ethical and scientific issues around the potential use of genome editing that might alter the genetics of future generations..$^{22}$

\section{References}

1. Jeon S, Lim JM, Lee HG, et al. Current status and perspectives of genome editing technology for microalgae. Biotechnol Biofuels 2017;10:267.

2. Strong A, Musunuru K. Genome editing in cardiovascular diseases. Nat Rev Cardiol 2017;14:11-20.

3. Gupta RM, Musunuru K. Expanding the genetic editing tool kit: ZFNs, TALENs, and CRISPR-Cas9. J Clin Invest 2014;124:4154-61.

4. Mao Z, Bozzella M, Seluanov A, Gorbunova V. DNA repair by nonhomologous end joining and homologous recombination during cell cycle in human cells. Cell Cycle 2008;7:2902-6.

5. Carroll D. Genome engineering with zinc-finger nucleases. Genetics 2011;188:773-82.

6. Sanjana NE, Cong L, Zhou Y, et al. A Transcription activator-like effector (TALE) toolbox for genome engineering. Nat Protoc 2012;7:171-92.

7. Zhang M, Wang F, Li S, et al. TALE: a tale of genome editing. Prog Biophys Mol Biol 2014;114:25-32.

8. Piatek A, Mahfouz MM. Targeted genome regulation via synthetic programmable transcriptional regulators. Crit Rev Biotechnol 2017;37:429-40.

9. Hsu PD, Lander ES, Zhang F. Development and applications of CRISPR-Cas9 for genome engineering. Cell 2014;157:1262-78.

10. Cong L, Ran FA, Cox D, et al. Multiplex genome engineering using CRISPR/Cas systems. Science 2013;339:819-23.

Figure 3. A single guide RNA (sgRNA), consisting of a crRNA sequence that is specific to the DNA target, and a tracrRNA sequence that interacts with the Cas? protein (1), binds to a recombinant form of Cas9 protein that has DNA endonuclease activity (2). The resulting complex will cause target-specific double-stranded DNA cleavage (3). The cleavage site will be repaired by the non-homologous end joining (NHEJ) DNA repair pathway, an error-prone process that may result in insertions/deletions (INDELs) that may disrupt gene function (4).
11. Zhang Q, Li Y, Zhao R, et al. The gainof-function mutation E76K in SHP2 promotes CAC tumorigenesis and induces EMT via the $\mathrm{Wnt} / \beta$-catenin sig- 
naling pathway. Mol Carcinog 2018;57:619-28.

12. Guay SP, Brisson D, Munger J, et al. ABCA1 gene promoter DNA methylation is associated with HDL particle profile and coronary artery disease in familial hypercholesterolemia. Epigenetics 2012;7:464-72.

13. Cox DB, Platt RJ, Zhang F. Therapeutic genome editing: prospects and challenges. Nat Med 2015;21:121-31.

14. Wang CX, Cannon PM. The clinical applications of genome editing in HIV. Blood 2016;127:2546-52.

15. Maeder ML, Gersbach CA. Genome- editing technologies for gene and cell therapy. Mol Ther 2016;24:430-46.

16. Yin H, Xue W, Chen S, et al. Genome editing with Cas9 in adult mice corrects a disease mutation and phenotype. Nat Biotechnol 2014;32:551-3.

17. Alanna S, Kiran M. Genome editing in cardiovascular diseases. Nature Rev Cardiol 2017;14:11-20.

18. Schulz R, Schlüter KD, Laufs U. Molecular and cellular function of the proprotein convertase subtilisin/kexin type 9 (PCSK9). Basic Res Cardiol 2015;110:4.

19. Lambert G, Sjouke B, Choque B, et al.
The PCSK9 decade. J Lipid Res 2012;53:2515-24.

20. Ding Q, Strong A, Patel KM, et al. Permanent alteration of PCSK9 with in vivo CRISPR-Cas9 genome editing. Circ Res 2014;115:488-92.

21. Gonçalves GAR, Paiva RMA. Gene therapy: advances, challenges and perspectives. Einstein (São Paulo) 2017;15:369-75.

22. Porteus MH. Towards a new era in medicine: therapeutic genome editing. Genome Biol 2015;16:286. 